\title{
Theranostics
}

2012; 2(10):1020-1036. doi: 10.7150/thno.4847

Review

\section{Light-Activated Content Release from Liposomes}

\author{
Sarah J. Leung and Marek Romanowski ${ }^{\bowtie}$ \\ Department of Biomedical Engineering, University of Arizona, Tucson, AZ 85721-0240, USA.
}

$\triangle$ Corresponding author: marekrom@email.arizona.edu.

(c) Ivyspring International Publisher. This is an open-access article distributed under the terms of the Creative Commons License (http://creativecommons.org/ licenses/by-nc-nd/3.0/). Reproduction is permitted for personal, noncommercial use, provided that the article is in whole, unmodified, and properly cited.

Received: 2012.07.19; Accepted: 2012.10.01; Published: 2012.10.18

\begin{abstract}
Successful integration of diagnostic and therapeutic actions at the level of individual cells requires new materials that combine biological compatibility with functional versatility. This review focuses on the development of liposome-based functional materials, where payload release is activated by light. Methods of sensitizing liposomes to light have progressed from the use of organic molecular moieties to the use of metallic plasmon resonant structures. This development has facilitated application of near infrared light for activation, which is preferred for its deep penetration and low phototoxicity in biological tissues. Presented mechanisms of light-activated liposomal content release enable precise in vitro manipulation of minute amounts of reagents, but their use in clinical diagnostic and therapeutic applications will require demonstration of safety and efficacy.
\end{abstract}

Key words: nanotechnology, nanomedicine, liposomes, drug delivery, plasmon resonance.

\section{Introduction}

Following the first reported preparation of liposomes, self-assembled vesicles having a structure evocative of the cell membrane [1], rhodopsin was reconstituted into such phospholipid vesicles and shown to initiate release of encapsulated cations upon exposure to blue-green light [2]. This release was attributed to increases in the membrane permeability brought about by the photoresponsive protein. A decade before the emergence of nanotechnology, this observation initiated the quest for biologically inspired nanometer-sized containers to enable manipulation of zeptoliters of solutions and yoctomoles of solutes.

Much of this work was done with the goal of producing robust methods for systemic delivery of therapeutic agents, to address problems of systemic toxicity, poor solubility in aqueous solvents, and insufficient stability in biological systems that are characteristic of many drug substances [3, 4]. Technological development of liposome-based drug delivery systems has reached a stage where stable encapsula- tion in liposomes can be achieved, even to a point where these non-covalent assemblies provide an encapsulation stability that may interfere with the efficient delivery of an active substance. In one particularly illustrative example, the lack of antitumor activity of certain liposomal formulation of cis-platin was attributed to its stable association with the liposome [5]. Thus, a release mechanism is required for a functional delivery system, and a research program succinctly formulated in the past is fully applicable today [6]:

"A considerable effort has been devoted to the development of liposomes for the transport and buffering of drugs in the body. Several research groups have reported the increased localization of sterically stabilized liposomes (PEG-liposomes) at tumor sites. If PEG-liposomes are to be effective carriers of therapeutic agents, their drug permeability must be sufficiently low that little passive release occurs during the circulation time of the PEG-liposomes. However, once PEG-liposomes reach tumor sites, it may be desirable 
to accelerate the release of the encapsulated drug. The use of light to stimulate the release of encapsulated compounds from liposomes is attractive, because it is possible to control the spatial and temporal delivery of the radiation."

There has been tremendous progress in the availability of light sources, that nowadays include broadly tunable laser systems capable of producing continuous illumination and pulses as short as femtoseconds, and multiphoton excitation can be routinely obtained at almost any wavelength. Great advances have been made in the area of plasmon resonant structures that can now be prepared with well controlled morphologies and user-defined optical properties. Furthermore, progress in microfluidics, 3-dimensional cell models, and highly sensitive detection technologies presents new areas for applications of controlled release systems that were not contemplated before. These advances directly impact progress in light-triggered liposomes in two ways. First, there are now new methods of imparting light sensitivity onto liposomes. These various mechanisms can generally be distinguished based on illumination mode, which may range from femtosecond pulses to continuous wave illumination. Some of these methods are simpler, safer, and more economically viable than earlier generations of light sensitive liposomes. Second, the demand for a controlled release mechanism reaches beyond drug delivery systems. The diversity of experimental approaches developed by research groups working in this area represents the ongoing efforts to tailor controlled release mechanisms to sometimes very specific applications.

This review focuses exclusively on methods for achieving release from liposomes using light. We include methods that can be broadly categorized as photochemical or photophysical. Release modalities within these categories are described in approximate chronological order of their development, with proposed mechanisms listed in Tables 1 and 2. A special focus within this review is on plasmon resonance-assisted release, an area of research with considerable activity in the last four or so years. Light-triggered release from vesicles other than liposomes (e.g., polymers, polyelectrolytes, micelles) is not discussed in this review. Similarly, research generally dealing with the interaction of light and lipid membranes, with no specific focus on content release, is not included here. Some of the broader aspects of research on controlled release are subject of excellent reviews [7 - 15]. A head-to-head comparison of the various release methods, with some universal metrics to describe the encapsulation efficiency, release rate, and biological safety and efficacy, would be ideal for such a review on light-induced release from liposomes. Unfortunately, reports in this area do not follow any single standard. Illumination conditions, including pulsed or continuous sources, collimated laser beams, or lamps with a broad spectral distribution, and measures of efficiency vary between methods. To preserve the verity of the original data, metrics are presented as reported by their authors.

\section{Photochemical activation of content re- lease}

Photochemical activation of content release from liposomes employs destabilization of the lipid membrane by light-induced isomerization, cleavage, or polymerization of its components (Table 1). These modalities often require that a lipid-like molecule with desired light-responsive properties be synthesized. Because of the energies involved in the chemical bonds of interest, the use of UV light is typically required, and strategies involving photosensitizing molecules responsive in visible and near infrared are sought.

Table I. Examples of methods of liposomal contents release by photochemical activation

\begin{tabular}{|c|c|c|c|}
\hline $\begin{array}{l}\text { Mechanism of } \\
\text { release }\end{array}$ & $\begin{array}{l}\text { Active } \\
\text { moiety }\end{array}$ & $\begin{array}{l}\text { Activation wave- } \\
\text { length }\end{array}$ & Reference \\
\hline \multirow{6}{*}{$\begin{array}{l}\text { photoisomeriza- } \\
\text { tion }\end{array}$} & azobenzene & UV & [16]. \\
\hline & & & [17] \\
\hline & & $\begin{array}{l}470 \mathrm{~nm} \text { with cho- } \\
\text { lesterol }\end{array}$ & {$[18,19]$} \\
\hline & retinoyl & UV & {$[20]$} \\
\hline & spiropyran & UV & [21] \\
\hline & stilbene & UV & {$[22]$} \\
\hline \multirow[t]{6}{*}{ photocleavage } & plasmalogen & $\begin{array}{l}630-820 \mathrm{~nm} \text {, sensi- } \\
\text { tized by phtalocy- } \\
\text { anines or bacterio- } \\
\text { chlorophyll }\end{array}$ & {$[23-25]$} \\
\hline & NVOC-DOPE & UV & {$[26]$} \\
\hline & dithiane & UV & {$[27,28]$} \\
\hline & o-nitrobenzyl & UV & [29] \\
\hline & coumarin & UV & {$[30]$} \\
\hline & $\begin{array}{l}\text { lipid double } \\
\text { bond }\end{array}$ & $\begin{array}{l}>580 \mathrm{~nm} \text {, sensitized } \\
\text { by phtalocyanines } \\
\text { or chlorin }\end{array}$ & {$[31]$} \\
\hline \multirow[t]{2}{*}{$\begin{array}{l}\text { photopolymeriza- } \\
\text { tion }\end{array}$} & bis-sorb PC & $\begin{array}{l}\text { UV } \\
>470 \mathrm{~nm} \text {, sensitized } \\
\text { by DiI }\end{array}$ & [32 - 37] \\
\hline & diacetylene PC & $\begin{array}{l}\text { UV } \\
514 \mathrm{~nm} \text {, sensitized } \\
\text { by payload }\end{array}$ & {$[38,39]$} \\
\hline
\end{tabular}


Table 2. Examples of methods of liposomal contents release by photophysical activation

\begin{tabular}{|c|c|c|c|}
\hline $\begin{array}{l}\text { Mechanism } \\
\text { of release }\end{array}$ & $\begin{array}{l}\text { Active } \\
\text { moiety }\end{array}$ & $\begin{array}{l}\text { Activation } \\
\text { wave- } \\
\text { length }\end{array}$ & $\begin{array}{l}\text { Refer- } \\
\text { ence }\end{array}$ \\
\hline \multirow[t]{5}{*}{$\begin{array}{l}\text { molecular } \\
\text { dyes }\end{array}$} & $\begin{array}{l}\text { calcein, aqueous core } \\
\text { hemoglobin, external }\end{array}$ & $\begin{array}{l}488 \mathrm{~nm} \\
577 \mathrm{~nm}\end{array}$ & {$[40,41]$} \\
\hline & sulforhodamine, aqueous core & $545 \mathrm{~nm}$ & [42] \\
\hline & ICG, external & $808 \mathrm{~nm}$ & {$[43,44]$} \\
\hline & tissue (hemoglobin), external & $527 \mathrm{~nm}$ & [45] \\
\hline & DiD, lipid membrane & $645 \mathrm{~nm}$ & {$[46-48]$} \\
\hline \multirow{11}{*}{$\begin{array}{l}\text { non-resona } \\
\text { nt } \\
\text { gold parti- } \\
\text { cles } \\
\text { plasmon } \\
\text { resonant } \\
\text { gold nano- } \\
\text { particles }\end{array}$} & $\begin{array}{l}\text { hydrophobic, } 2.5 \mathrm{~nm} \text { dia } \\
\text { aqueous core, } 2.8 \mathrm{~nm} \text { dia } \\
\text { lipid-metal complex, } 1.4 \mathrm{~nm} \text { dia }\end{array}$ & UV & [49] \\
\hline & hydrophobic, 5nm dia & UV & [50] \\
\hline & $\begin{array}{l}\text { gold-coated liposomes, } \\
\text { degradable to }<6 \mathrm{~nm} \text { dia. }\end{array}$ & $\begin{array}{l}\text { tunable, } \\
650-1100 \mathrm{n} \\
\mathrm{m}\end{array}$ & $\begin{array}{l}{[54,57,} \\
58]\end{array}$ \\
\hline & $\begin{array}{l}\text { hollow gold nanospheres, } \\
33 \mathrm{~nm} \text { dia., tethered }\end{array}$ & $820 \mathrm{~nm}$ & [59] \\
\hline & $\begin{array}{l}\text { solid gold spheres, } 20 \mathrm{~nm} \text { dia., } \\
\text { electrostatic aggregates }\end{array}$ & $650 \mathrm{~nm}$ & [60] \\
\hline & $\begin{array}{l}\text { solid gold spheres, } 80 \mathrm{~nm} \text { dia., } \\
\text { aqueous core }\end{array}$ & $520 \mathrm{~nm}$ & [61- 64] \\
\hline & $\begin{array}{l}\text { gold coated liposomes } \\
10,20,70 \mathrm{~nm} \text { dia. }\end{array}$ & $\begin{array}{l}\text { tunable, } \\
600-750 \\
\mathrm{~nm}\end{array}$ & [63] \\
\hline & $\begin{array}{l}\text { gold-coated liposomes } \\
238 \mathrm{~nm} \text { dia. }\end{array}$ & $808 \mathrm{~nm}$ & [64] \\
\hline & $\begin{array}{l}\text { solid gold spheres, } 13 \mathrm{~nm} \text { dia., } \\
\text { tethered }\end{array}$ & $520 \mathrm{~nm}$ & [65] \\
\hline & $\begin{array}{l}\text { solid gold spheres, } 50 \mathrm{~nm} \text { dia., } \\
\text { tethered }\end{array}$ & $520 \mathrm{~nm}$ & [66] \\
\hline & $\begin{array}{l}\text { gold nanorods, } \\
\text { co-localization with liposomes }\end{array}$ & $808 \mathrm{~nm}$ & [67] \\
\hline
\end{tabular}

\section{I. Photoisomerization}

Release of encapsulated content from liposomes by photoisomerization is typically achieved by embedding a molecule that experiences a conformational change upon light illumination into the bilayer membrane; the conformational change destabilizes the lipid membrane and allows for release. Photoisomerizable moieties most frequently used for light-controlled release of liposomal contents are based on azobenzene. Upon ultraviolet (UV) illumination, azobenzene isomerizes from an extended trans form to a twisted cis form, and returns back to the trans form under blue light or heat (Figure 1a). Both the steric effect of the cis form and the increased polarity of this conformation can potentially destabilize the lipid bilayer. An early demonstration of this principle comes from the Kunitake lab, who described preparation of a photoresponsive lipid membrane [16] by including a synthetic single chain amphiphatic azobenzene conjugate, $\mathrm{C}_{4} \mathrm{Azo}$, into a 1,2-dipalmitoyl-sn-phosphatidylcholine (DPPC)based lipid vesicle, 1:5 mol ratio, obtained by sonication. The trans-cis photoisomerization of the azobenzene was induced by a 5 minute illumination with $360 \mathrm{~nm}$ light from a $500 \mathrm{~W}$ high pressure $\mathrm{Hg}$ lamp. Perturbation of the membrane structure of these 25-30 $\mathrm{nm}$ vesicles increased liposomal membrane permeability to water entrapped inside the vesicles and to bromothymol blue entrapped within the lipid bilayer.

The azobenzene moiety is a basis of a synthetic photochromic phospholipid that was designed and extensively studied by Morgan and coworkers [17]. Bis-azo PC (1,2-bis(4- $n$-butylphenylazo-4'phenylbutyroyl)-L-a-phosphatidylcholine) resembles DPPC and contains two photochromic acyl chains (Figure 1a). This synthetic phospholipid was used to construct large unilamellar vesicles that encapsulated fluorescent calcein to monitor content release. Optimization of liposome composition and release conditions produced a system where solute release could be achieved on a millisecond time scale upon laser illumination using $8 \mathrm{~ns}$ pulses from a $2 \mathrm{~mm}$-diameter beam of $355 \mathrm{~nm}$ light producing $15 \mathrm{~mJ} /$ pulse [18]. It was also shown that the addition of up to $25 \mathrm{~mol} \%$ cholesterol to the liposomal membrane caused the liposomes to release their contents in response to visible light in the $470 \mathrm{~nm}$ region, to which liposomes lacking steroid are insensitive [19].

Photoisomerizable liposome compositions have also been prepared using retinoyl-phospholipids [20]. Lipid vesicles of 50-300 nm diameter were made entirely of diretynoylphosphatidylcholine, and full release of encapsulated carboxyfluorescein was accomplished within $30 \mathrm{~s}$ of irradiation at $360 \mathrm{~nm}$ and 50 $\mathrm{W} / \mathrm{m}^{2}$ in the presence of oxygen. The authors noted the reduced effect of illumination on membrane permeability under oxygen-free conditions, which may indicate the role of photooxidation of retinoic acid in permeabilization of this bilayer membrane.

Liposomal content release was also accomplished by photoisomerization of spiropyran [21]. Spiropyran was covalently attached to the hydrophobic terminus of a single chain lipid, and this conjugate was then incorporated into DPPC-based liposomes at up to $10 \%$. Photoisomerization of hydrophobic spiropyran using $365 \mathrm{~nm}$ illumination yielded hydrophilic merocyanine and destabilized the membrane. Other photoisomerizable amphiphiles tested in light-induced liposomal content release include 4-dodecyl-4'-(3-phosphate-trimethyleneoxy)azobenze ne and 4-hexylstilbene-4'-butyric acid [22]. 
a

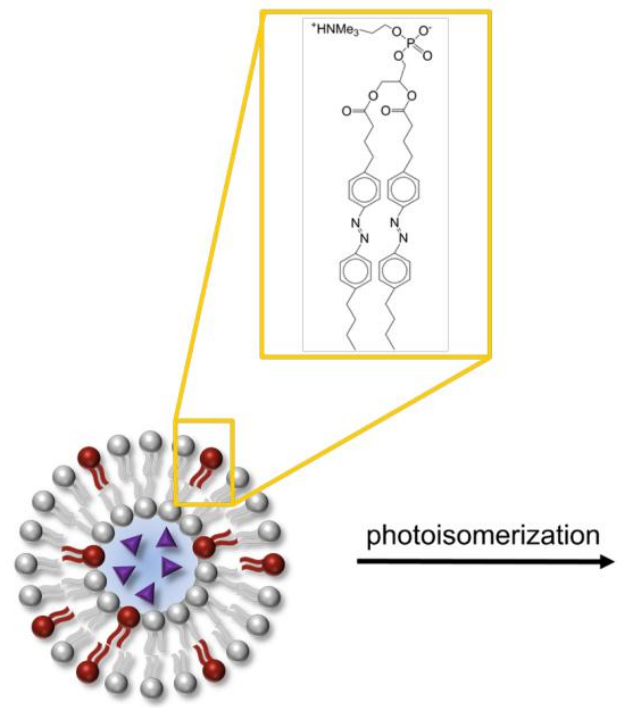

b

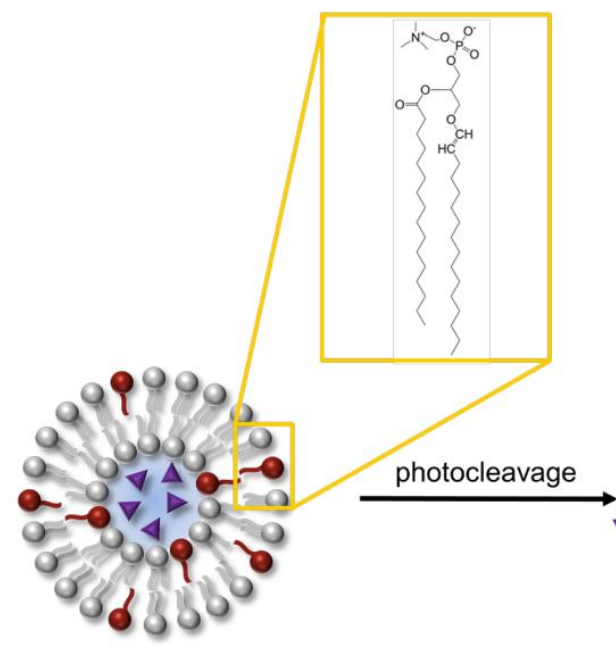

C

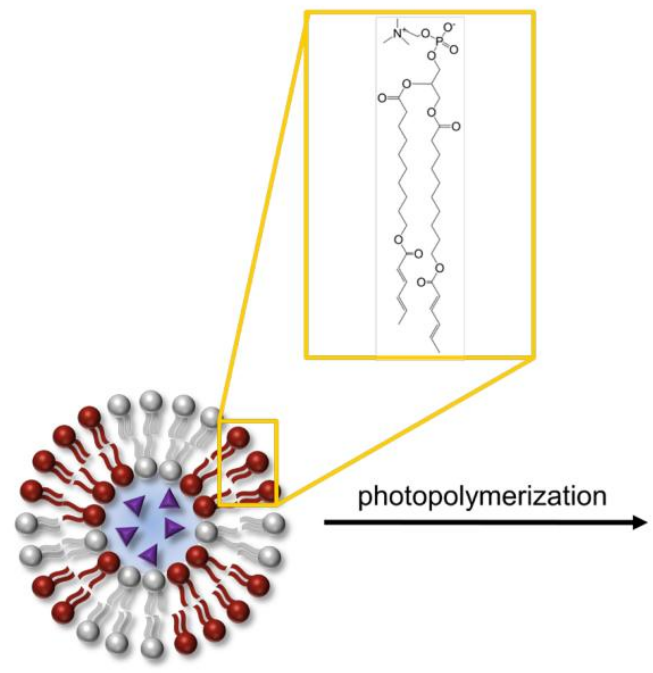

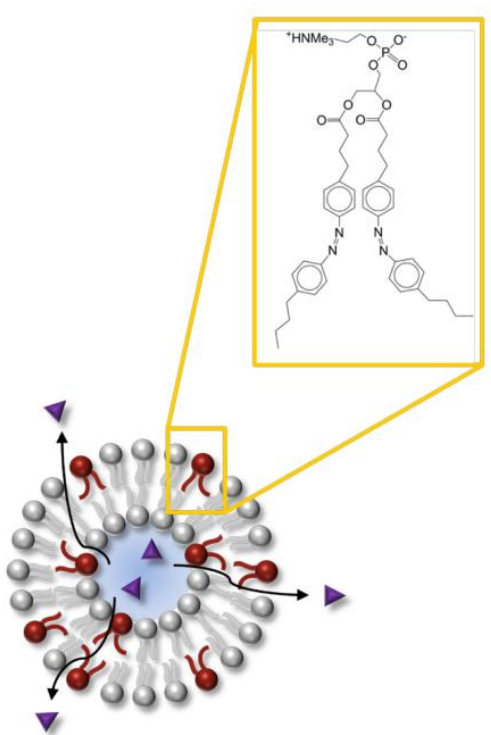
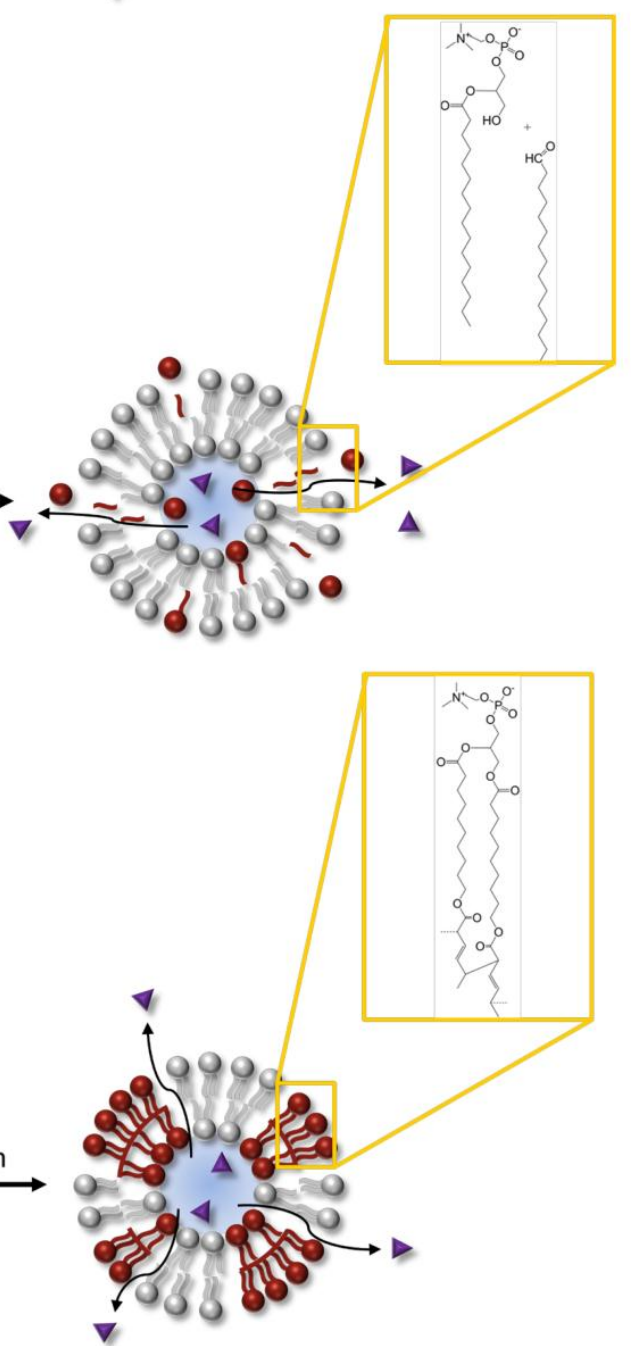

Figure I. Light activated photochemical release from liposomes. Photochemical release can be achieved via photoisomerization (a), photocleavage (b), or photopolymerization (c). In photoisomerization, azobenzene included in the acyl chains of lipids isomerizes from an extended trans form to a twisted cis form. Light-induced fragmentation of plasminogen causes a loss of amphiphilic character. Photopolymerization of bis-sorbPC leads to the formation of lipid domains. These processes all lead to destabilization of the liposome bilayer and release of encapsulated contents. 
Efficient release from liposomes using photoisomerization has been achieved using amphiphilic molecules that structurally resemble or are derived from membrane-forming lipids. The structural similarity between these light-sensitive molecules and the liposomal membrane-forming lipids may improve the stable encapsulation of a payload under non-illumination conditions. Subsequent release rates of encapsulated content in response to illumination appear to be responsive to the bulk liposome composition, indicating that, while photoisomerization is the critical trigger for destabilization, the extent of that membrane destabilization and the resulting increase in its permeability depends on its bulk composition. However, despite demonstration of fairly rapid release, the main obstacle to the development of medical applications based on photoisomerization remains the fact the these processes require illumination at short wavelengths, which is associated with limited penetration depth and potential phototoxicity within biological samples.

\subsection{Photocleavage}

Controlled release by photocleavage typically involves inclusion of some form of photocleavable amphiphilic molecule into the liposomal membrane; subsequent illumination of these modified liposomes triggers separation of the polar and nonpolar moieties of this molecule, with a concomitant loss of amphiphilic character and resulting in destabilization of the liposome.

A broad strategy for content release by photocleavable lipids was introduced by Thompson and coworkers [23]. This strategy is based on photo-induced cleavage of naturally occurring lipids called plasmalogens by photodynamic sensitization. In this design, $100 \mathrm{~nm}$ diameter lipid vesicles were made from $80 \%$ or more plasmalogen in combination with auxiliary lipids, such as lecithin, polyethylene glycol (PEG)-lipid, or cholesterol. Photoinduced cleavage of the labile $s n-1$ vinyl ether linkage of plasmalogen resulted in vesicle leakage and was exploited to trigger delivery (as depicted in Figure 1b). The photocleavage is sensitized by molecules generally incorporated in the hydrophobic region of the bilayer. In a series of experiments, plasmalogen fragmentation was sensitized by zinc phthalocyanine, tin octabutoxyphthalocyanine, or bacteriochlorophyll a; these components absorb light between 630 and 820 $\mathrm{nm}$ and made triggering of liposomal content release by red and near infrared light possible. Bacteriochlorophyll a sensitization produced the fastest release rate from liposomes: $100 \%$ calcein was released in less than $20 \mathrm{~min}$ (using $800 \mathrm{~nm}$ light at $300 \mathrm{~mW}$ ); this rate was two orders of magnitude faster than that for non-sensitized egg lecithin liposomes under identical experimental conditions [24]. A cascade of liposomal triggering was also demonstrated using $800 \mathrm{~nm}$ light to trigger release of $\mathrm{Ca}^{2+}$, which then activated PLA2-mediated release of contents from non-photosensitive DPPC vesicles [25].

A photocleavable lipid derived from 1,2-dioleoyl-sn-phosphatidylcholine (DOPE), called NVOC-DOPE, was also shown to be capable of forming stable liposomes [26]. Irradiation of liposomes comprising this lipid using a $150 \mathrm{~W}$ xenon lamp produced fragmentation of that derivative (having an absorption maximum at $345 \mathrm{~nm}$ ) to separate 3,4-dimethoxy-2-nitrosobenzaldehyde from DOPE. This formation of DOPE, a lipid that does not form a bilayer structure under physiological conditions, destabilized the liposome membrane and induced calcein release. Up to $50 \%$ release was achieved after 40 min of irradiation.

Another photocleavable lipid has been synthesized by linking hydrophilic phosphocholine headgroups to hydrophobic tails via a photolabile, dithiane-based tether [27, 28]. Subsequently, $100 \mathrm{~nm}$ diameter liposomes were prepared from a mixture of 1-palmitoyl-2-oleoyl-sn-phosphatidylcholine

(POPC):cholesterol:photolabile lipid in the ratio 5:3:2. These liposomes were shown to unload encapsulated pyridine upon UV irradiation. Photocleavage of this lipid, which has a maximum absorbance at $350 \mathrm{~nm}$, results in loss of amphiphilicity, destabilization of the liposome, and release of contents. The release was analyzed by comparing the lifetime of encapsulation. While the dark leakage (non-irradiated) lifetime was on the order of $20 \mathrm{~h}$, irradiation of the sample at wavelengths longer than $300 \mathrm{~nm}$ using a filtered mercury UV lamp for 75 min decreased the lifetime to $1.5 \mathrm{~h}$.

Photocleavable amphiphilic molecules prepared by connecting nonpolar stearyl amine with charged amino acids, such as glutamic acid (Glu), aspartic acid (Asp), and lysine (Lys), via their o-nitrobenzyl derivatives [29] were combined with liposomes prepared using 1,2-distearoyl-sn-phosphatidylcholine (DSPC) as a major component (95 $\mathrm{mol} \%)$ and the individual photocleavable lipids as minor components (5 mol\%). Carboxyfluorescein was loaded within the liposomal core as a self-quenching marker of encapsulation and release. The proposed mechanism of photo-triggered release involved hydrolysis of o-nitrobenzyl upon illumination with UV light and subsequent destabilization of the liposomal membrane. This release mechanism turned out to be relatively slow. In the case of the Glu-based photocleavable amphiphile, 
approximately $60 \%$ content was released after 2 hrs illumination using a $100 \mathrm{~W}$ UV lamp producing 365 nm light.

Photocleavable amphiphiles have also been synthesized using the coumarin chromophore to link a nonpolar heptadecyl chain to a polar headgroup: Glu, Lys or ethylenediamine [30]. Liposomes were prepared using $20 \mathrm{~mol} \%$ of cleavable lipids with $80 \%$ of diacylphosphatidyl choline. Content release triggered by illumination with $254 \mathrm{~nm}$ light for 2 hrs was more effective when unsaturated lipids were included in the liposome composition. The efficiency of release varied with the type of the headgroup, with the least effective being Glu and the most effective being ethylenediamine.

The search for a release method triggered by long wavelength illumination led to the investigation of photosensitizers for photodynamic permeabilization of liposome membranes [31]. Exposure of various liposome compositions to red light in the presence of photosensitizers, such as aluminum trisulfophthalocyanine, chlorin, or neutral zinc phthalocyanine, was tested. In these experiments, liposomes in the presence of a photosensitizer were exposed for 1 minute to light from a xenon lamp $\left(0.4 \mathrm{~W} / \mathrm{m}^{2}\right.$ thorough a 580 $\mathrm{nm}$ long pass filter). Light triggered carboxyfluorescein release, presumably due to oxidative damage to acyl chains, reached $90 \%$ in 10 minutes or longer time. Liposomes made of fully saturated lipids did not exhibit leakage upon photodynamic action, as their oxidation was likely substantially hindered. Furthermore, sodium azide, a singlet oxygen quencher, considerably reduced light-induced content leakage, confirming the proposed photodynamic mechanism [31].

In contrast to photoisomerization, the photocleavage approach led to examples where content release was achieved using more deeply penetrating and biologically safe light wavelengths. The use of dyes absorbing in the red to NIR range to sensitize plasmalogen fragmentation appears to be the most successful photocleavage strategy from the perspective of biological applications, combining safer delivery with more efficient release. Unfortunately, this advantage is typically at the expense of formation of deleterious reactive oxygen species produced by photosensitizers.

\subsection{Photopolymerization}

The photo-induced crosslinking of lipids is another photochemical mechanism for inducing release from liposomes. The O'Brien group found that cross-linking polymerization of bis-substituted lipids in the presence of unreactive lipids causes the for- mation of lipid domains [32]. Subsequently, it was shown that UV-induced crosslinking of lipids could effectively destabilize PEGylated liposomes [33]. The UV-induced cross-linking polymerization of 1,2-bis[10-(2', 4'-hexadienoyloxy)-decanoyl]-sn-phosp hatidylcholine (bis-sorbPC) in liposomes comprising cholesterol, 1,2-dioleoyl-sn-phosphatidylcholine (DOPC), and PEG2000-DOPE, caused an over 100 -fold increase in the permeability of an encapsulated fluorescent marker (illustrated in Figure 1c). Furthermore, the addition of cyanine dyes to these polymerizable liposomes rendered them sensitive to the wavelengths of light absorbed by the dye [34]. A particularly effective photosensitizer was found to be green light absorbing 1,1'-dioctadecyl-3,3,3',3'tetramethylindocarbocyanine iodide (DiI), a lipophilic dye. This photosensitizer was used to achieve visible light-induced destabilization of bis-sorbPCcontaining PEG-liposomes and release of pyrene-1,3,6,8-tetrasulfonic acid (PTSA), a fluorescent marker [35]. This mechanism of light triggered release was applied to these same polymerizable liposomes following their endocytosis by HeLa cells [36]. Using an encapsulated $\mathrm{pH}$-sensitive marker, 8-hydroxypyrene-1,3,6-trisulfonic acid (HPTS), it was shown that photolysis of endocytosed liposomes moves their contents from areas of low $\mathrm{pH}$ to those of high $\mathrm{pH}$, indicating delivery of the marker from the endosome to cytosol. Further development of the polymerizable liposomes led to a chiefly saturated liposome composition, PEG-DSPE/ 1,2-diarachidoylsn-phosphatidylcholine (DAPC)/bis-sorbPC, that exhibited extremely low permeabilities to water-soluble fluorescent probes at $37^{\circ} \mathrm{C}$ in the absence of light, yet increased its permeability 28,000-fold upon UV irradiation [37].

A different type of photopolymerizable liposome was prepared from DPPC and diacetylene phospholipid, 1,2-bis(tricosa-10,12-diynoyl)-sn-phosphatidylcholine $\left(\mathrm{DC}_{8,9} \mathrm{PC}\right)$ and was shown to efficiently released entrapped calcein upon $254 \mathrm{~nm}$ illumination [38]. To advance these formulations toward in vivo applications, triggering using visible light and the effect of released anticancer drugs on cellular toxicity were tested. Sonicated liposomes containing various ratios of DPPC: $\mathrm{DC}_{8,9} \mathrm{PC}$ and $4 \mathrm{~mol} \%$ DSPE-PEG2000 were loaded with calcein (excitation and emission at 485 and $517 \mathrm{~nm}$, respectively) or a chemotherapeutic drug, doxorubicin (excitation and emission at 490 and $590 \mathrm{~nm}$, respectively). Liposomes containing 10 or 20 mol\% $\mathrm{DC}_{8,9} \mathrm{PC}$ and illuminated with $514 \mathrm{~nm}$ laser light for 1-3 min released calcein or doxorubicin in a wavelength-specific manner. This observation suggested that visible light-induced solute leakage from 
the liposomes depended on the spectral properties of entrapped solutes, rather than those of the lipid membrane. It was concluded that this release occurs via an alternate mechanism, unrelated to photopolymerization. Laser treatment of co-cultures containing doxorubicin (DOX)-loaded liposomes and cells resulted in at least a 2-3 fold improved cell killing as compared to untreated samples [39].

Similarly to photocleavage, successful photopolymerization of lipids and liposomal content release was achieved using safer wavelengths of light than those used for photoisomerization. In contrast to photocleavage, this method exhibits greater increases in liposomal permeability to encapsulated solutes and subsequently more rapid rates of release. Like the photoisomerization mechanisms discussed earlier, these release rates appear to correlate to the bulk liposome composition rather than the method of triggering release. The use of photopolymerization has made drastic steps towards the application of photochemistry to the controlled release from liposomes, yet the question of biodegradability of the polymerized carrier has not been addressed.

\section{Photophysical activation of content re- lease}

Photophysical release from liposomes does not rely on any chemical changes of structures within or associated with the bilayer membrane. Examples of photophysical release discussed here take advantage of photothermal conversion of absorbed light with ensuing thermal and/or mechanical processes in the lipid membrane and the surrounding medium. The methods for achieving photophysical release are developed around various light-absorbing moieties: molecular dyes, metallic particles, and plasmon resonant gold nanoparticles (Table 2).

\section{I. Molecular Absorbers}

Of the many competing paths of excited state deactivation in molecular dyes, photothermal conversion is especially efficient in dyes that are non-fluorescent or that have their fluorescence quenched, e.g., through the concentration effect. There are several arrangements of liposomes with dyes acting as a photothermal transducer. A hydrophilic dye can be encapsulated in the aqueous interior of the liposome (Figure 2a), a hydrophobic dye or lipid-dye conjugate can be incorporated in the lipid membrane (Figure 2b), or a liposome can be surrounded by a solution of dye. As with many molecular dyes in biological applications, the primary challenges that need to be addressed are photobleaching, limited availability of dyes with strong absorption in the near infrared range, and possible photochemical processes. However, this straightforward design of photothermal heating via molecular absorbers has led to several in vivo demonstrations of light controlled content release from liposomes.

A series of reports concerned with ophthalmic drug delivery systems established the feasibility of laser induced content release by photothermal heating. This was accomplished through photothermal heating of a dye encapsulated within the liposomes or by photothermal heating of surrounding tissue [40]. The release of encapsulated fluorescent calcein from the aqueous core of $250 \mathrm{~nm}$ diameter temperature sensitive liposomes made of DPPC and 1,2-dipalmitoyl-sn phosphatidylglycerol (DPPG) was tested in whole blood and buffered solution. Using an argon and a dye laser operating between 20 and 80 $\mathrm{mW}$, illumination was provided at $488 \mathrm{~nm}$, at the absorption maximum of calcein, or at $577 \mathrm{~nm}$, near the maximal absorption of hemoglobin. In buffer, release of content was achieved by $488 \mathrm{~nm}$ but not at $577 \mathrm{~nm}$, demonstrating photothermal release mediated by encapsulated fluorescein. However, in whole blood nearly complete release was achieved upon either 488 $\mathrm{nm}$ or $577 \mathrm{~nm}$ illumination, indicating efficient photothermal conversion by hemoglobin. Hemoglobin-mediated photothermal effect was then implicated in subsequent in vivo adenosine diphosphate delivery for therapeutic occlusion of retinal vessels reported by the same group [41]. In vivo content release required power densities of approximately 10 $\mathrm{W} / \mathrm{cm}^{2}$ delivered in 1 second pulses from the blue-green argon laser.

Liposomal release of sulforhodamine (Figure 3) by localized heating has been shown using single nano- or picosecond laser pulses [42]. Liposomes were made with 90\% DPPC in two sizes, $2 \mu \mathrm{m}$ and $4.5 \mu \mathrm{m}$ diameter. The method of lipid rehydration chosen by the authors yields primarily multilamellar lipid vesicles, generally of larger size and lower encapsulation capacity than unilamellar vesicles. Encapsulation of sulforhodamine at concentrations of $1-50 \mathrm{mM}$ resulted in dimerization of the fluorescent molecule, with concomitant shift in absorption maximum (from $585 \mathrm{~nm}$ to $545 \mathrm{~nm}$ ) and self-quenching. Most excitation energy at $532 \mathrm{~nm}$ is deactivated by non-radiative pathways, with the quenching efficiency estimated at $98 \%$. Using 8 ns pulses, nearly complete release was achieved at a $50 \mathrm{mM}$ dye concentration and $0.5 \mathrm{~J} / \mathrm{cm}^{2}$ energy density. Using 25 ps pulses, it required 5 times less energy to obtain comparable dye release. It was noted that ps pulses improved release efficiency by depositing thermal energy within the liposome, with negligible heat loss due to diffusion over the pulse 
duration.

a

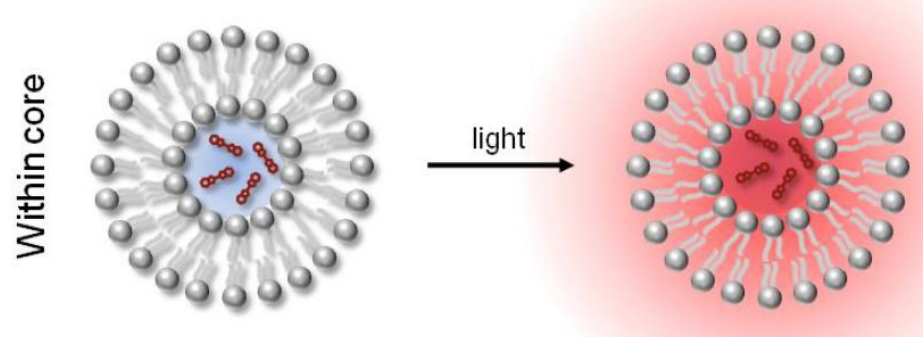

b

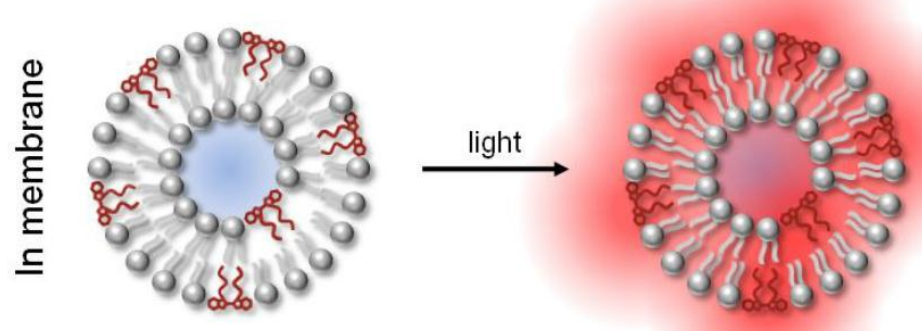

Figure 2. Release from liposomes mediated by molecular absorbers. Hydrophilic molecular absorbers may be included in the liposomal core (a) and hydrophobic absorbers in the bilayer. Upon illumination, molecular absorbers mediate photothermal conversion, which may increase membrane permeability of thermosensitive liposomes.<smiles>O=[S+]([O-])c1cc(S(=O)(=O)O)ccc1C1=C2C=C3C4=[N+](CCC2)CCCC4=C1Oc1cc2c4c(c13)CCCN4CCC2</smiles>

Indocyanine green

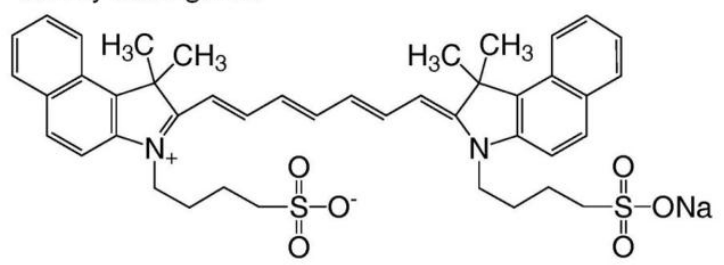

1,1'-dioctadecyl-3,3,3',3'-tetramethylindodicarbocyanine iodide<smiles>CCN1c2ccccc2C(C)(C)C1CCC=CC1=[N+](CC)c2ccccc2C1(C)C</smiles>

Figure 3. Structures of molecular absorbers used to achieve photothermal release from liposomes. 
Dye-mediated photothermal release has been applied towards studies of circulation and thermal damage in laser photocoagulation procedures [43]. Here, a micellar form of indocyanine green (ICG), molecular structure shown in Figure 3, was injected to enhance the photothermal effect of laser irradiation at $808 \mathrm{~nm}$ (continuous wave). Then, liposomes containing self-quenching concentrations of carboxyfluorescein were injected. Carboxyfluorescein was selected to avoid direct absorption of laser light. In this two-component system, photothermal conversion in ICG produced thermal lysing of liposomes and rapid release of the fluorescent dye into vasculature. Varied laser fluences, from 60 to above $190 \mathrm{~J} / \mathrm{cm}^{2}$, produced distinct fluorescence patterns indicative of clearance, extravasation, and coagulation. One of the proposed applications of this type of laser-induced release of carboxyfluorescein was quantification of the thermal damage incurred in the process of laser coagulation. This release mechanism was successfully tested in this and other models in vivo [44].

More recently, release from liposomes by photothermal conversion within surrounding tissue was shown in transgenic reporter mice that constitutively express luciferase throughout the body [45]. The luciferin-luciferase reaction provided readout in living cells and tissues. Since this reaction is limited to the cell cytoplasm, the bioluminescent signal indicates extracellular release of luciferin from the liposomes, followed by cellular uptake. Liposomes made of 1-palmitoyl-2-hydroxy-sn-phosphatidylcholine

(MPPC):DPPC:DSPE-PEG2000 and having a $76 \mathrm{~nm}$ diameter were selected for their reasonable in vivo half-life and good thermal release kinetics at $42{ }^{\circ} \mathrm{C}$. Illumination for 2 minutes using an Nd:YLF laser operating at $527 \mathrm{~nm}$ and $650 \mathrm{~mW}$ average power led to release of luciferin from the intravenously injected liposomes. As shown by subsequent thermal and luminescence imaging in vivo, the release was localized within the target tissue by using laser light to transiently heat the region of the skin surface to $45^{\circ} \mathrm{C}$.

Liposomes can be sensitized to light by embedding lipophilic dyes in the bilayer membrane of the liposomes [46]. Both one-photon and two photon photolysis of liposomes have been shown, enabling the use of far red to near infrared laser wavelengths for release. In follow-up studies, mass spectrometry analysis and insensitivity of the process to radical inhibitors confirmed the photophysical nature of release [47]. Subsequently, dye-sensitized lipid nanocapsules were used to deliver a cell-impermeable molecule, a ubiquitous second messenger inositol trisphosphate $\left(\mathrm{IP}_{3}\right)$, into cells following endocytosis. In these experiments, $100 \mathrm{~nm}$ diameter lipid vesicles composed of 9:1 DPPC:DOPC and incorporating a small fraction of 1,1'-dioctadecyl-3,3,3',3'tetramethylindodicarbocyanine iodide (DiD), shown in Figure 3, were photolyzed with a single $645 \mathrm{~nm}, 3$ ns pulse at $600 \mathrm{~nJ} /$ pulse; ensuing release of $\mathrm{IP}_{3}$ in the cytosol was evident by $\mathrm{Ca}^{2+}$ concentration changes as monitored by a calcium sensitive dye [48]. This method demonstrated light-addressable release of content, whereby the activation by light can be accomplished using red or even near infrared wavelengths in a manner independent of spectral properties of the encapsulated content and surrounding medium.

Incorporation of molecular dyes into lipid vesicles offers the ability to sensitize cargo release to a wide array of light wavelengths, making many of these approaches applicable for in vivo delivery. It has also been employed to achieve rapid release rates, but typically in conjunction with high laser powers. Nevertheless, these rapid release techniques utilizing two photon photolysis represent attractive possibilities for in vitro studies, particularly in cellular signaling, due to their high temporal precision.

\subsection{Nonresonant gold nanoparticles}

Very small nanoparticles of gold, in the single nanometer size range, have been shown to mediate photothermal conversion in liposomes, leading to release of contents [49]. This has been shown in three types of gold-liposome configurations: hexanethiol-capped hydrophobic gold nanoparticles incorporated in the liposome bilayer (Figure 4a), gold nanoparticles protected with mercaptosuccinic acid and carrying negative charge encapsulated in the aqueous liposomal core (Figure 4b), and gold nanoparticles covalently linked to the polar lipid headgroups (Figure 4c). Liposomes of 200-500 $\mathrm{nm}$ diameter were prepared using DSPC:DPPC (90:10) by reverse phase evaporation, with calcein as a fluorescent marker of encapsulation and release. The incorporated small particles of gold were no larger than $2-3 \mathrm{~nm}$ in diameter, did not exhibit a plasmon resonance band, and had Rayleigh extinctions monotonically increasing toward shorter wavelengths. Therefore, photothermal release was elicited by illumination at $250 \mathrm{~nm}$. The light-mediated release process from these liposomes required approximately $30 \mathrm{~min}$ to reach $60-80 \%$ content release, depending on the type of gold association. These release rates were 100 fold greater than those found for plain liposomes.

This strategy based on non-resonant photothermal conversion in small gold nanoparticles was later applied to demonstrate the release of a biologically relevant alakoid, berberine [50]. In that work, 
hydrophobic gold nanoparticles of $5 \mathrm{~nm}$ diameter were embedded in the bilayer membrane of thermosensitive liposomes prepared by the supercritical $\mathrm{CO}_{2}$ method. Release was achieved by exposing these preparations to $250 \mathrm{~nm}$ light. The initial kinetics of release was faster than previously demonstrated, with over $50 \%$ release achieved in $5 \mathrm{~min}$.

The main limitation of these early approaches is, perhaps, that they use gold particles of sizes that do not support plasmon resonance. Therefore, photothermal conversion necessitated the use of UV light, where absorption efficiency is low, required illumination times are long, and phototoxicity potential is high. On the other hand, the small size of nanoparticles used would be preferred for clinical use, as these sizes are generally compatible with renal clearance.

\subsection{Toward lower phototoxicity - Plasmon resonance-assisted release}

The hallmark of plasmon resonance in metallic nanoparticles is the intense absorption and scattering of electromagnetic radiation occurring, in the Rayleigh approximation, when $\varepsilon=-2 \varepsilon_{m}$, where $\varepsilon$ is the dielectric function of the particles and $\varepsilon_{m}$ is that of the medium. The refractive index of gold closely meets the plasmon resonance conditions near $520-530 \mathrm{~nm}$, resulting in the characteristic red color of suspensions of gold nanoparticles. However, further control of the spectral position of this resonance is possible by varying particle shape and size, leading to tunability of optical properties of such particles throughout the visible to near-infrared range. This tunability opens opportunities for applications employing light wavelengths that are safe and that penetrate biological tissues to significant depths. The spectral properties of metallic nanoparticles of different shapes were explained by Gustav Mie in the early 1900's [51], although experimental challenges prevented the practical realization of fully controllable optical properties in such materials until much later in the $20^{\text {th }}$ century. In one particularly illustrative example, Aden and Kerker's computational analysis based on the Mie theory showed that the spectral position of plasmon resonances generated by a concentric metallic shell on a dielectric core depends on the ratio of the inner-to-outer diameter of that shell [52]. In general, increasing the amount of gold and, therefore, the thickness of gold shell deposited onto the surface of a dielectric core shifts the spectral position of the resonance maximum toward shorter wavelengths (Figure 5). Experimental demonstration of this concept was later provided by seed-mediated deposition of gold onto the surface of silica nanoparticles [53].
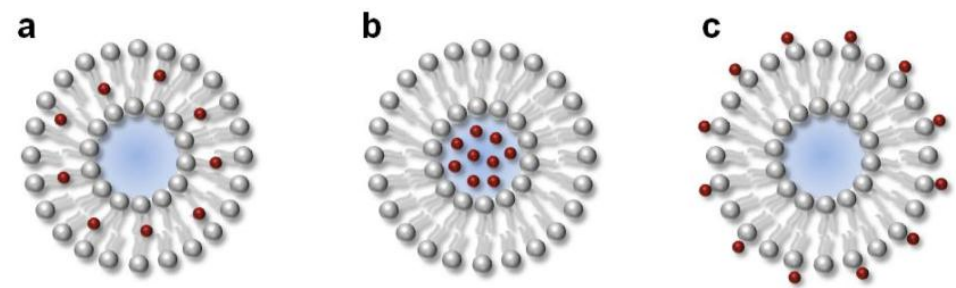

Figure 4. Light release from liposomes mediated by nonresonant gold nanoparticles. Nonresonant nanoparticles can be combined with liposomes by embedding them within the bilayer (a), encapsulating them within the core (b), or attaching them to the surface (c).

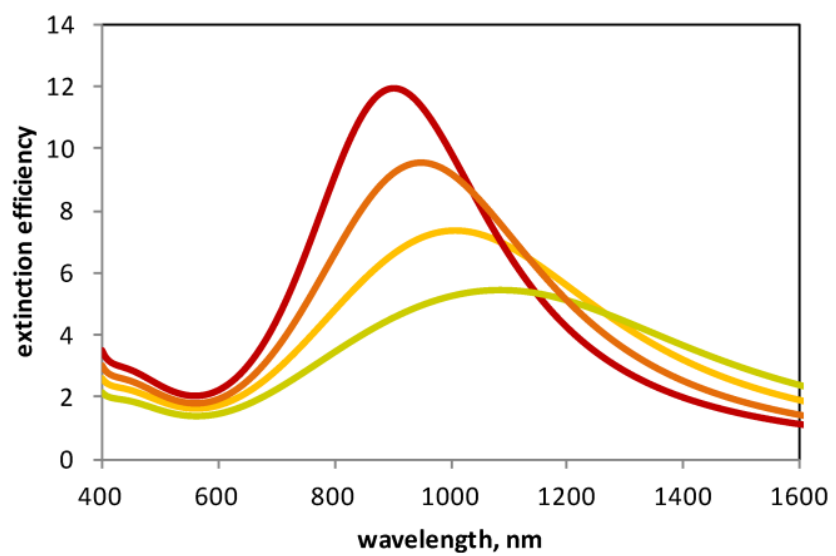

Figure 5. Calculated extinction spectra of plasmon resonant gold nanoshells. Plasmon resonance peaks correspond to gold nanoshells with a $100 \mathrm{~nm}$ core and having 2, 2.5, 3, $3.5 \mathrm{~nm}$ thickness (green, yellow, orange and red, respectively). The plasmon resonance of gold shells is tunable according to shell thickness, with thicker shells exhibiting resonances at shorter wavelengths. 
In the first demonstration of plasmon resonance-assisted release from liposomes, our laboratory accomplished similar spectral tunability in a structure touted as gold-coated liposomes [54]. In that design, thermosensitive liposomes made with DPPC, a monoacyl phospholipid, and a PEG-lipid conjugate were coated with gold clusters by in situ reduction of gold chloride (Figure 6). This process was derived from the method of lipid vesicle metallization through the formation of zero-valent metal-lipid complexes described earlier [55]. Formation of plasmon resonance in these gold-coated liposomes was evident by the emergence of extinction spectrum with extinction peaks that could be varied in a broad range, extending from $650-1100 \mathrm{~nm}$, by changing the amount of ionic gold added to the liposomal surface.

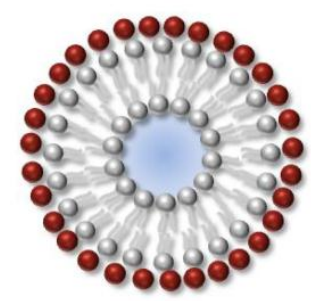

Figure 6. Degradable plasmon resonant gold-coated liposomes. These composite structures comprise $100 \mathrm{~nm}$ diameter liposomes surrounded by a discontinuous shell of gold nanoclusters having diameters in the range of single nanometers. The use of a liposome with discrete gold clusters renders these shells degradable and potentially enables their clinical use.

As highly efficient resonance is obtained in metallic particles having diameters in tens of nanometers, use of plasmon resonant nanoparticles for in vivo applications raises concerns regarding clearance mechanisms of particles this size from the body, and unknown long term effects of particle accumulation in tissues. Recently proposed criteria of nanoparticles' clinical utility include degradability to components clearable by renal filtration, i.e., to components having a hydrodynamic diameter of 5-6 nm or less [56], generally smaller than the diameter of a typical plasmon resonant structure of gold. In a manner that addresses the need for both efficient light absorption and degradability, the plasmon resonant structure of gold-coated liposomes is composed of an array of gold clusters that, upon degradation, yield components of a clearable size. Dimensional stability and degradability of this composite material is provided by the liposome template. The spectrally tunable optical properties of this composite shell can be ex- plained by approximating its dielectric constant $\varepsilon_{s}$ using the Maxwell Garnet effective medium theory $[54,57]$ :

$$
\varepsilon_{s}=\varepsilon_{m} \frac{\varepsilon_{A u}(1+2 f)+\varepsilon_{m}(1-f)}{\varepsilon_{A u}(1-f)+\varepsilon_{m}(2+f)}
$$

where $f$ is the fill factor, i.e., the ratio of the volume of the gold clusters to the volume of the composite shell, $\varepsilon_{A u}$ is the size-dependent dielectric function of gold nanoparticles, and $\varepsilon_{m}$ is the dielectric constant of the suspension medium (water). The overall volume of the gold clusters, hence the fill factor $f$, is controlled by varying the amount of tetrachloroaureate ions added to the suspension of liposomes; therefore, $\varepsilon_{s}$ can be controlled experimentally. In the manner explained by the Mie theory, by varying $\varepsilon_{s}$ in this shell-like structure, optical resonances at different wavelengths can be produced, leading to fill factor-based optical tunability. Increasing the fill factor by adding more gold produces resonances at longer wavelengths (Figure 7), which is in accordance with experimental observation.

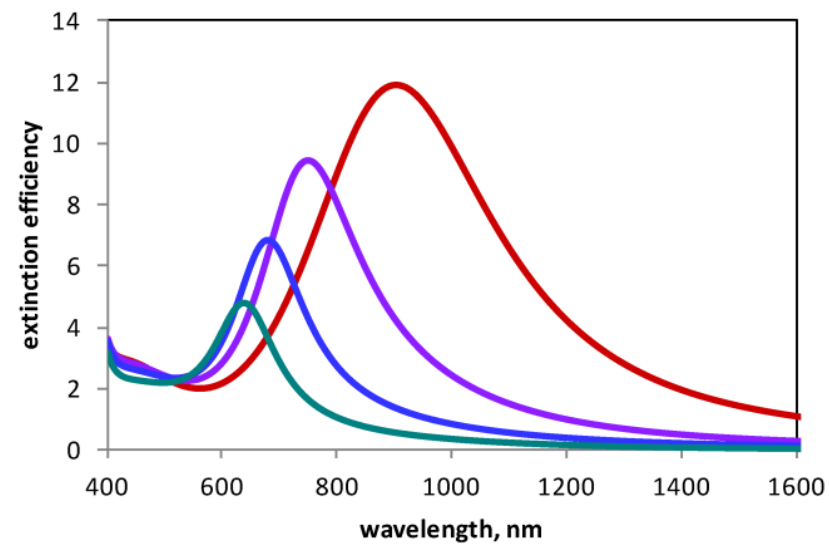

Figure 7. Calculated extinction spectra of gold-coated liposomes. Plasmon resonance peaks correspond to $100 \mathrm{~nm}$ liposomes coated with $2 \mathrm{~nm}$ gold particles with the fill factor of $0.7,0.8,0.9$, and 1.0 (teal, blue, purple, and red, respectively). Longer resonances are achieved by covering the surface of $100 \mathrm{~nm}$ diameter liposomes more densely with gold clusters. Note that the red curve describes a solid shell $(2 \mathrm{~nm}$ thick, $100 \mathrm{~nm}$ core, fill factor of I) and is identical with the red curve in Figure 4. The computational model utilizes the Mie scattering theory and the Maxwell Garnett effective medium theory and is described elsewhere [58].

Light-induced release from these plasmon resonant structures is similar to that from liposomes modified with molecular absorbers (Section 3.1). When illuminated with laser light, temperature rise due to photothermal conversion at the gold shell resulted in increased permeability of liposomal mem- 
brane and release of an encapsulated fluorescent marker. As similarly shown using molecular dyes for photothermal heating, the use of a pulsed laser beam to elicit release potentially enables high spatial resolution of this process. Quantitatively, this analysis is often presented in terms of thermal confinement. Laser energy absorbed by a metallic particle diffuses into the surrounding medium. This process is controlled by thermal diffusivity for water, $k=0.143 \times 10^{-6} \mathrm{~m}^{2} / \mathrm{s}$. The time required for three-dimensional diffusion of heat over a distance $\mathrm{r}$ can be estimated as $t=r^{2} / 6 k$. For example, to confine thermal changes to $r=10 \mu \mathrm{m}$, laser pulses of $0.1 \mathrm{~ms}$ or shorter are required. At the same time, release of molecules from liposomes by the photothermal conversion mechanism relies on the diffusion of molecules across the bilayer. With a typical diffusion constant of a molecule in the bilayer membrane of $D=1 \times 10^{-14} \mathrm{~m}^{2} / \mathrm{s}$ and a membrane thickness of $d=4 \mathrm{~nm}$, the time required to cross the membrane is $t=d^{2} / 2 D=0.4 \mathrm{~ms}$. One striking conclusion from this analysis is that it should be impossible to elicit photothermal content release localized to the volume of one cell using a single sub-millisecond pulse of laser light. However, sequences of short pulses, in the $\mu$ s range, have been devised to achieve superb spatial control of the release process $[57,58]$.

A fundamentally different mechanism of liposomal content release introduced by the Zasadzinski lab involves a form of photoacoustic conversion [59]. In this design, hollow gold nanoparticles have a $33 \mathrm{~nm}$ diameter and $820 \mathrm{~nm}$ absorption peak were synthesized and encapsulated within liposomes or tethered to the outside of liposomes through a PEG linker (Figure 8a). Liposome disruption and leakage of content was triggered by irradiation with 130 fs pulses from a Ti:sapphire laser operating at $800 \mathrm{~nm}$ and 1 $\mathrm{kHz}$. The gradual disappearance of the $820 \mathrm{~nm}$ absorption peak with a concomitant increase in $520 \mathrm{~nm}$ absorption suggests that hollow gold nanospheres are melted in the process of release. However, the temperature of the bulk solution increases by just $1{ }^{\circ} \mathrm{C}$, which alone is not sufficient for thermal release of liposome content. This in conjunction with the fact that no release was observed under continuous wave illumination suggested that a pressure wave-based mechanism induced release. a

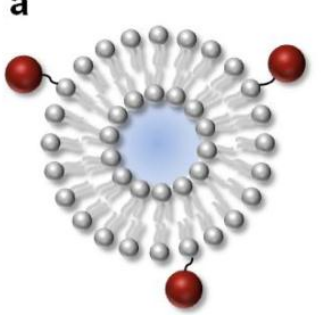

b

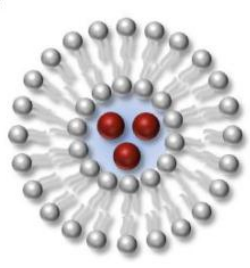

C

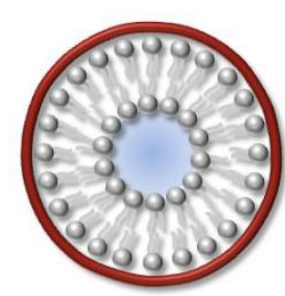

Figure 8. Light mediated photomechanical release using plasmon resonance. Plasmon resonant structures can be tethered to the outside of liposomes (a), placed within the liposomal core (b), or created using the liposome as a template (c). Photothermal conversion in these structures leads to release of encapsulated content via membrane disruption by thermal or mechanical stress.

Using the very short laser pulses described in that report, the temperature increase at the gold nanoshells causes localized thermal expansion, or mechanical stress, within the sample [59]. Noteworthy, the mechanical stress is considered confined to the illuminated volume, a sphere of diameter $d$, as long as the laser pulse duration $t<d / v$, where $v$ is the velocity of sound. Considering a typical liposome of $d$ $=0.1 \times 10^{-6} \mathrm{~m}$ and the velocity of the pressure wave in water at $v=1,484 \mathrm{~m} / \mathrm{s}$, this condition of pressure confinement is obtained at pulses shorter than 67 ps. This stress will then dissipate as a pressure wave propagating in the suspension medium with energy falling as $1 / r^{2}$. This shockwave effect is highly localized and only the liposomes adjacent to illuminated nanoshells (through encapsulation or tethering) release content upon pulsed illumination, leading to exquisite spatial control of release. Furthermore, this release mechanism is fairly general and independent of the thermal properties of liposomal membranes, and may be applied to permeate a range of membrane compositions, including cellular membrane.

In another demonstration of plasmon resonance-mediated release from liposomes Volodkin et al. [60] used solid gold nanoparticles and their aggregates under near infrared laser illumination. Here, citrate stabilized gold nanoparticles $20 \mathrm{~nm}$ diameter and cationic liposomes made of DPPC, 1,2-dipalmitoyl-3-trimethylammonium propane (DPTAP), and cholesterol, approximately $100 \mathrm{~nm}$ in diameter were used to form electrostatic complexes. Due to their hydrophilic nature and large size, the particles were not integrated into the lipid bilayer. Rather, gold nanoparticles were aggregated by salt, so 
that their collective plasmon resonance shifted to longer wavelengths as desired. Photothermal content release was induced by $50 \mathrm{~mW}$ illumination from an $830 \mathrm{~nm}$ diode laser for the total of around $5 \mathrm{~s}$. The release from these liposomes was mediated either by heating of the lipid membrane to a temperature above the main phase transition of DPPC, the chief component of the membrane, or by disruption of the membrane due to vapor bubble formation. The described system for achieving content release has a remarkable simplicity. The size of nanoparticles and their aggregates, the use of cationic lipids to promote formation of complexes, and the strong dependence on electrostatic interaction make this system suitable for researchers developing in vitro models, whereas those interested in development of clinical applications may find implementation of this system rather challenging.

The Lapotko group [61-64] investigates formation of vapor bubbles around plasmon resonant particle illuminated with intense ns pulses of laser light. The use of ns pulses makes this approach distinctly different from the condition of stress confinement associated with ps and shorter pulses [59]. At the same time, formation of vapor bubbles makes this mechanism different from photothermally activated leakage [55]. Formation of such bubble results in rapid ejection of liposome contents, faster than that based on photothermal heating with subsequent diffusion. Experiments were carried out using cationic liposomes of $1 \mu \mathrm{m}$ diameter, comprising $80 \%$ DOPC and $20 \%$ 1,2-dioleoyl-3-trimethylammonium-propane (DOTAP). Gold nanoparticles of $80 \mathrm{~nm}$ diameter were incorporated within the liposomal core (Figure 8b). Formation of plasmonic nanobubbles was observed when the liposome-entrapped gold nanoparticles were illuminated using a single $0.5 \mathrm{~ns}$ laser pulse at $532 \mathrm{~nm}$. A bubble of vaporized medium formed over the pulse duration, with the maximal bubble diameter controlled by pulse energy. The plasmonic bubble lifetimes in the range of 10-1000 ns and sizes varying from $50 \mathrm{~nm}$ to micrometers may be achieved. The rapidly expanding bubble mechanically disrupts liposomes and releases their content. This technology can potentially be used to permeabilize a wide range of capsules, from liposomes to entire cells.

Several variants of the gold-coated liposomes introduced by our group have been developed. In one example, liposomes were coated with a solid shell of gold (Figure 8c), where shell growth was directed by a layer of poly-L-histidine located on the liposome surface, capable of chelating metal ions [65]. The plasmon resonance bands of these 10,20 , and $70 \mathrm{~nm}$ diameter liposome supported shells were centered at approxi- mately 600, 690, and $750 \mathrm{~nm}$. These appear to be solid gold shells, and the stability of these capsules is defined by the gold shell rather than the original liposome, alleviating the potential problem of content leakage at the room temperature. Incorporation and release of carboxyfluorescein and doxorubicin was shown. Notably, release from these solid shells required $10-20 \mathrm{~mJ} / \mathrm{cm}^{2}$. This amount of energy is significant, in the sense that the amount of laser illumination required to elicit release results in gold shell deformation or melting, as observed by electron microscopy, converting these into larger aggregates of gold. The design of large shells and resulting aggregates do not address requirements for clinical use, but yields structures whose stability does not rely on the presence of water. Therefore, these gold shells, capable of carrying biological agents, can be freely manipulated and stored in a manner not possible with plain liposomes.

In yet another demonstration of gold-coated liposomes, $200 \mathrm{~nm}$ diameter lipid vesicles of soybean lecithin and cholesterol were prepared in distilled water and coated by a silica layer [66]. The resulting silica shell was then coated with gold by in situ reduction of gold chloride with hydroxylamine. The authors report that these capsules were incubated with doxorubicin, which resulted in incorporation of the drug within the capsules. Release of the content was accomplished by illumination with an $808 \mathrm{~nm}$ laser beam at $100 \mathrm{~mW}$ for $15 \mathrm{~min}$. When tested in cancer cell cultures, laser irradiation of these composite vesicles, blank or carrying doxorubicin, resulted in approximately $58 \%$ and $82 \%$ cell growth inhibition, respectively.

Similarly, several variants of the nanoparticle-liposome tethering method (Figure 8a) have been described. In one example, tethering was accomplished using complementary DNA sequences [67]. Liposomes of approximately $100 \mathrm{~nm}$ diameter and made of DPPC included a small fraction of DOPE-N-(4-(p-maleimidophenyl)butyramide) (MPBPE) (5\% by weight), to which a thiol-modified DNA was conjugated. In a separate preparation, $13 \mathrm{~nm}$ gold nanoparticles were functionalized using another complementary strain of thiol-terminated DNA. Attachment of gold to liposome surfaces was then accomplished by combining these complementary sequences of DNA. Depending of the length of complementary DNA sequences, gold nanoparticles were either $8 \mathrm{~nm}$ apart or in direct contact with the surface of liposomes. Upon light exposure, liposomes with gold nanoparticles in direct contact with their surface released their encapsulated calcein, whereas liposomes with the $8 \mathrm{~nm}$ spacer did not. The gold particles 
used here exhibit plasmon resonance at $530 \mathrm{~nm}$, a typical position for particles of this size, and their assembly at the surface of the liposomes resulted in spectral shifts toward longer wavelengths. However, the authors chose to use UV light to initiate photothermal conversion, and release was rather slow, reaching no more than $15 \%$ over $100 \mathrm{~min}$, in a manner consistent with earlier observations using UV light with gold nanoparticles to elicit liposomal release [49].

In another example of the tethering approach, the stability of $100 \mathrm{~nm}$ diameter liposomes was first improved by cross-linking the diacetylene lipid chains [68]. The partial polymerization of the liposomal membrane, evident by the absorption peak at $443 \mathrm{~nm}$ and orange sample color, prevented calcein leakage, even at $40^{\circ} \mathrm{C}$, for three days. Subsequently, $50 \mathrm{~nm}$ diameter particles of gold were tethered to liposomes via a lipid functionalized with 1,2-dithiolane. Using just ten $6 \mathrm{~ns}$ pulses of a $532 \mathrm{~nm}$ laser beam operating at $10 \mathrm{~Hz}$ frequency and up to $400 \mathrm{~mJ} /$ pulse resulted in $70 \%$ content release from these tethered conjugates. To demonstrate the in vitro efficacy of this delivery system, doxorubicin was entrapped in these capsules. These doxorubicin-gold-liposomes were incubated with a breast cancer cell line and, after 12 hrs, fluorescence microscopy showed cytoplasmic localization of doxorubicin. Following $532 \mathrm{~nm}$ illumination, these cells maintained $80 \%$ viability. To achieve significant reduction in viability, these doxorubicin-loaded capsules were illuminated before their addition to cells; incubation with pre-illuminated doxorubicin-goldliposomes reduced viability to approximately $20 \%$.

In contrast to these examples where gold and liposomes are linked to form light-activated release systems, plasmon resonance-assisted liposomal content release has been accomplished in vivo via co-injection of plasmon resonant gold nanorods and liposomes loaded with doxorubicin [69]. Here, thermosensitive $100 \mathrm{~nm}$ liposomes made of DPPC:DMPC:cholesterol:DSPE-PEG2000 were prepared and loaded with doxorubicin by the ammonium sulfate gradient method. Gold nanorods were synthesized by the seed-mediated growth method and coated with thiolated methoxy-PEG, MW 5000. Liposomes with doxorubin and gold nanorods were not linked, but co-injected intravenously in a mouse xenograft model of human glioblastoma; after $48 \mathrm{hrs,}$ the tumor site was illuminated with an $808 \mathrm{~nm}$ near infrared laser operating at $0.5 \mathrm{~W} / \mathrm{cm}^{2}$ to elicit content release. Gold nanorod-mediated release was highly effective in suppressing tumor progression. Stable encapsulation of doxorubicin within liposomes in circulation for durations over $48 \mathrm{hrs}$, enhanced per- meation and retention of these liposomes at the tumor xenograft site, and a localized photothermal temperature increase to $43^{\circ} \mathrm{C}$ mediated by gold nanorods contributed to this successful in vivo demonstration of remotely controlled content release.

The advantage offered by tunable plasmon resonant structures is two-fold. First, the resonant enhancement of absorption efficiency produces required photophysical responses for release from much lower energy densities, or fluences. Second, the spectral position of resonance can be set at wavelengths optimal for both tissue penetration and operation of a particular laser system. Together, plasmon resonance yields an efficient release mechanism of potentially low phototoxicity. However, as described previously, a drawback of these structures is that this efficient plasmon resonance is obtained in metallic particles having diameters in tens of nanometers. This raises concerns regarding lack of degradability, nonexisting clearance mechanisms of particles this size from the body, and unknown long term effects of particle accumulation in tissues. Continued development of degradable plasmon resonant structures will overcome this obstacle to in vivo use.

\section{Summary}

Development of technology for light-controlled release initially focused on photochemical processes. Despite successful demonstrations of these release mechanisms, there are several obstacles on the path to the clinical utility of photochemical modalities. Relatively high energies involved in photochemistry necessitate the use of UV light, associated with concerns regarding UV radiation photoxicity and its effect on the stability of biological systems and the subsequent safety of this method in medical applications. While considerable efforts have been devoted to the development of photosensitizing strategies, the increasing complexity of such systems and formation of reactive oxygen species associated with many photosensitizers will likely prevent broad medical use of photochemically-controlled liposomal content release.

Successfully tested photophysical methods for releasing liposomal contents involve some form of photothermal conversion, using either molecular dyes or plasmon resonant gold nanoparticles placed within or in proximity to lipid membranes. Molecular dyes sought for this application generally suffer from the problems often discussed in the context of fluorescent probes for optical imaging: wavelength choice in the near infrared is limited, molecules are subject to photobleaching, and, perhaps most importantly, the excited states of dyes may promote formation of reactive species. On the other hand, the availability of certain 
dyes and their conjugates, particularly cyanine and xanthene derivatives, may facilitate development of dyes having desired photophysical and safety properties, and offers reasonable prospects for regulatory approval. One of the most promising developments in the dye-mediated controlled release of liposomal content is the demonstration of two-photon release via photothermal conversion in lipophilic cyanine conjugates incorporated into the membrane [48]. Pulsed illumination in the near infrared range produced instantaneous and full content release, without formation of free radicals. Delivery of focused laser pulses required for two photon processes may create a technological challenge worth addressing in the path toward demonstrating the clinical feasibility of this modality.

Recent advances in preparation of plasmon resonant structures has resulted in materials exhibiting exceptionally strong absorption in the visible and near infrared range, with the added advantage of continuous tunability of spectral characteristics, which allows for optimization with both laser source and target environment, be it a tissue or microreactor. Plasmon resonant enhancement of absorption is required for reaching a rate of release that is of practical clinical use. However, the size of metallic nanoparticles necessary for strong and tunable plasmon resonances, which is on the order of tens of nanometers, raises questions regarding the long term effects of accumulation of these particles in the body, including unknown toxicity and interference with other diagnostic and therapeutic modalities. Compatibility of plasmon resonant materials with long term patient survival has yet to be demonstrated. The reported development of degradable plasmon resonant structures may address these requirements [58], and demonstration of stability and degradation of these materials in vivo would likely provide momentum for the discussion of the many clinical uses of this controlled delivery and release mechanism.

\section{Outlook}

While there are many technologies for achieving light-controlled content release from liposomes, it is apparent that none of these is currently fully compatible with clinical use. Two broad research directions emerge from this situation. In one, a substantial effort needs to be applied toward guiding these technologies onto the path to clinical development. In vivo demonstration of safety, stability and release from degradable gold-coated liposomes or two-photon activated release using appropriate molecular dyes exemplify this direction. In another, these technologies have a great and immediate potential for controlling miniscule amounts of materials for applications in the laboratory. Highly sensitive diagnostic assays, single cell manipulations, microfluidic devices, synthetic biology, and femtoliter chemistry [70 75], exemplify applications that could benefit from these controlled release modalities, with ongoing research possibly offering further refinement of the spatiotemporal control of such experiments. One example of such a research direction deals with optical trap based manipulation of gold coated liposomes currently investigated our laboratory [76]. The main premise of this approach is that the high polarizability of the plasmon resonant gold coating, responsible for efficient absorption of light, also generates a strong gradient force in a highly focused beam of laser light. Optical trapping of these gold coated liposomes may therefore be possible with relatively low intensities of laser light.

The main medical need addressed by these controlled release technologies is the delivery of therapeutic agents with unprecedented spatial and temporal control and low systemic exposure. In cancer chemotherapy, the ability to deliver high concentrations of cytotoxic agents while minimizing their systemic exposure may improve the therapeutic index of the drug and produce more efficacious treatment. Considering that over $90 \%$ of cancers originate in the epithelium $[77,78]$ and the depth of epithelium is well within the penetration range of near infrared light [79], a standard argument holds that near-infrared light is especially well-suited for controlled delivery of therapeutic substances. However, most cancer-related mortality is the result of metastatic disease [80], and accessibility to the primary tumor may have little impact on improving the prognosis of advanced disease. Therefore, the true potential of this technology is linked to methods for early disease diagnosis, so that the primary cancer may be detected and destroyed before spreading to other tissues that are less accessible to these controlled release modalities.

Combination of diagnostic and therapeutic modalities, or theranostics some may say, presents quite a challenging set of requirements. Considering a systemic route delivery, efficient therapy requires a sustained high concentration of drug that is localized to the diseased site. Requirements for molecular diagnostic imaging are essentially opposite, and include a full volume distribution of agents with fairly quick elimination to yield a comprehensive diagnostic image with low background. The versatile liposome-based delivery platform combined with exquisite control of content release may help address these disparate requirements. Conjugation of targeting ligands to liposomes in any of the controlled release 
modalities described here would impart a molecular targeting function onto these nanocapsules. Many of the liposomal carriers described here already have linkers appropriate for such modifications, and all of them can potentially have such a linker introduced as a lipid-PEG conjugate. Adding molecular targeting and testing the complete functionality of such a light-controlled release system represents an important step toward the development of a combined diagnostic and therapeutic modality.

While efficient photothermal conversion alone can be used to destroy targeted cancerous epithelial cells, there is growing evidence that selective killing of epithelial cancer cells can actually promote proliferative signals generated by adjacent cancer associated fibroblasts, or can activate proliferative signals from cancer stem cells [81, 82]. Either of these pathways may lead to cancer relapse. Therefore, better understanding and management of communication pathways acting in the tumor microenvironment becomes an important paradigm in developing effective cancer treatments. Further development of light-controlled release technology may allow for the delivery of agents that act upon a cancer cell and its complex microenvironment, destroying both the cell and signaling pathways supporting cancer. Possible clinical implementations may include the delivery of chemotherapy agents to augment precise tumor excision, to maximize cancer removal while preserving the functional status of the patient. Specific examples of clinical opportunities that may warrant further investigation are improved cytoreduction in ovarian cancer, localized chemotherapy to supplement glioma resections, or new treatment options for cutaneous lymphomas.

\section{Acknowledgements}

This work was supported in part by grants from the National Institutes of Health (CA120350 and HL007955) and the National Science Foundation (CBET 0853921).

\section{Competing Interests}

The authors have declared that no competing interest exists.

\section{References}

1. Bangham AD, Horne RW. Negative staining of phospholipids and their structural modification by surface-active agents as observed in the electron microscope. J Mol Biol. 1964; 8: 660-668.

2. O'Brien DF, Zumbulyadis N, Michaels FM, Ott RA. Light-regulated permeability of rhodopsin:eggphosphatidylcholine recombinant membranes. Proc Natl Acad Sci USA. 1977; 74: 5222-5226.

3. Chabner BA, Roberts TG. Chemotherapy and the war on cancer. Nat Rev Cancer 2005; 5: 65-72.

4. Waring MJ. The search for new anticancer drugs. Lancaster, UK: Kluwer Academic Publishers; 1992.
5. Zamboni WC, Gervais AC, Egorin MJ, Schellens JHM, Zuhowski EG, Pluim D, Joseph E, Hamburger DR, Working PK, Colbern G, Tonda ME, Potter DM, Eiseman JL. Systemic and tumor disposition of platinum after administration of cisplatin or STEALTH liposomal-cisplatin formulations (SPI-077and SPI-077 B103) in a preclinical tumor model of melanoma. Cancer Chemother Pharmacol. 2004; 53: 329-336.

6. Boundurant B, Mueller A, O'Brien DF. Photoinitiated destabilization of sterically stabilized liposomes. Biochim Biophys Acta. 2001; 1511: 113-122.

7. Gerasimov OV, Boomer JA, Qualls MM, Thompson DH. Cytosolic drug delivery using $\mathrm{pH}$ - and light-sensitive liposomes. Adv Drug Deliv Rev. 1999; 38: 317-338.

8. Shum P, Kim JM, Thompson DH. Phototriggering of liposomal drug delivery systems. Adv Drug Deliv Rev. 2001; 53: 273-284.

9. Andresen TL, Jensen SS, Jørgensen K. Advanced strategies in liposomal cancer therapy: Problems and prospects of active and tumor specific drug release. Prog Lipid Res. 2005; 44: 68-97.

10. Alvarez-Lorenzo C, Bromberg L, Concheiro A. Light-sensitive intelligent drug delivery systems. Photochem Photobiol. 2009; 85: 848-860.

11. Yavlovich A, Smith B, Gupta K, Blumenthal R, Puri A. Light-sensitive lipid-based nanoparticles for drug delivery: design principles and future considerations for biological applications. Mol Membrane Biol. 2010; 27: 364-381.

12. Katz JS, Jason A. Burdick JA. Light-responsive biomaterials: Development and applications. Macromol Biosci. 2010; 10: 339-348.

13. Alkilany AM, Murphy CJ. Toxicity and cellular uptake of gold nanoparticles: what we have learned so far? J Nanopart Res. 2010; 12: 2313-2333.

14. Timko BP, Whitehead K, Gao W, Kohane DS, Farokhzad O, Anderson D, Langer R. Advances in drug delivery. Annu Rev Mater Res. 2011; 41: $1-20$.

15. Zasadzinski JA, Wong B, Forbes N, Braun G, Wu G. Novel methods of enhanced retention in and rapid, targeted release from liposomes. Curr Opin Colloid Interface Sci. 2011; 16: 203-214.

16. Kano K, Tanaka Y, Ogawa T, Shimomura M, Kunitake T. Photoresponsive artificial membrane. Regulation of membrane permeability of liposomal membrane by photoreversible cis-trans isomerization of azobenzene. Photochem Photobiol. 1981; 34: 323 - 329.

17. Morgan CG, Thomas EW, Sandhu SS, Yianni YP, Mitchell AC. Light-induced fusion of liposomes with release of trapped marker dye is sensitised by photochromic phospholipid. Biochim Biophys Acta. 1987; 903: 504-509.

18. Bisby RH, Mead C, Morgan CG. Photosensitive liposomes as 'cages' for laser-triggered solute delivery: the effect of bilayer cholesterol on kinetics of solute release. FEBS Letters 1999; 463: 165-168.

19. Bisby RH, Mead C, Morgan CG. Wavelength-programmed solute release from photosensitive liposomes. Biochem Biophys Res Comm. 2000; 276: 169-173.

20. Pidgeon C, Hunt CA. Light sensitive liposomes. Photochem Photobiol. 1983; 37: $491-494$.

21. Ohya Y, Okuyama Y, Fukunaga A, Ouchi T. Photo-sensitive lipid membrane perturbationby a single chain lipid having terminal spiropyran group. Supramolec Sci. 1998; 5: 21-29.

22. Lei Y, Hurst JK. Photoregulated potassium ion permeation through dihexadecyl phosphate bilayers containing azobenzene and stilbene surfactants. Langmuir. 1999; 15: 3424-3429.

23. Anderson VC, Thompson DH. Triggered release of hydrophilic agents from plasmalogen liposomes using visible light or acid. Biochim Biophys Acta. 1992; 1119: 33-42.

24. Thompson DH, Gerasimov OV, Wheeler JJ, YuanjinRui Y, Anderson VC. Triggerable plasmalogen liposomes: improvement of system efficiency. Biochim Biophys Acta, 1996; 1279: 25-34.

25. Wymer NJ, Gerasimov OV, Thompson DH. Cascade liposomal triggering: Light-induced $\mathrm{Ca}^{2+}$ release from diplasmenylcholine liposomes triggers PLA2-catalyzed hydrolysisand contents leakage from DPPC liposomes. Bioconj Chem. 1998; 9: 305-308.

26. Zhang ZY, Smith BD. Synthesis and characterization of NVOC-DOPE, a caged photoactivatable derivative of dioleoylphosphatidylethanolamine. Bioconjugate Chem. 1999; 10: 1150-1152.

27. Wan Y, Angleson JK, Kutateladze AG. Liposomes from novel photolabile phospholipids: Light-induced unloading of small molecules as monitored by PFG NMR. J Am Chem Soc. 2002; 124: 5610-5611.

28. Li Z, Wan Y, Kutateladze AG. 2003. Dithiane-based photolabileamphiphiles: toward photolabile liposomes. Langmuir. 2003; 19:6381-6391.

29. Chandra B, Subramaniam R, Mallik S, Srivastava DK. Formulation of photocleavable liposomes and the mechanism of their content release. Org Biomol Chem. 2006; 4: 1730-1740. 
30. Subramaniam R, Xiao Y, Li Y, Qian SY, Sun W, Mallik S. Light-mediated and H-bond facilitated liposomal release: the role of lipid head groups in release efficiency. Tetrahedron Lett. 2010; 51: 529-532.

31. Pashkovskaya A, Kotova E, Zorlu Y, Dumoulin F, Ahsen V, Agapov I, Antonenko Y. Light-triggered liposomal release: Membrane permeabilization by photodynamic action. Langmuir 2010; 26: 5726-5733.

32. O'Brien DF, Armitage B, Benedicto A, Bennett DE, Lamparski HG, Lee YS, Srisiri W, Sisson TM. Polymerization of preformed self-organized assemblies. Acc Chem Res. 1998; 31: 861-868.

33. Bondurant B, O'Brien DF. Photoinduced destabilization of sterically stabilized liposomes. J Am Chem Soc.1998; 120: 13541-13542.

34. Clapp PJ, Armitage BA, O'Brien DF. Two-dimensional polymerization of lipid bilayers: Visible-light-sensitized photoinitiation. Macromolecules. 1997; 30: 32-41.

35. Mueller A, Bondurant B, O'Brien DF. Visible-light-stimulated destabilization of PEG-liposomes. Macromolecules. 2000; 33: 4799-4804.

36. Miller CR, Clapp PJ, O'Brien DF. Visible light-induced destabilization of endocytosed liposomes. FEBS Lett. 2000; 467: 52-56.

37. Spratt T, Bondurant B, O'Brien DF. Rapid release of liposomal contents upon photoinitiated destabilization with UV exposure. Biochim Biophys Acta. 2003; 1611: 35-43.

38. Yavlovich A, Singh A, Tarasov S, Capala J, Blumenthal R, Puri A. Design of liposomes containing photopolymerizable phospholipids for triggered release of contents. J Therm Anal Calorim. 2009; 98: 97-104.

39. Yavlovich A, Singh A, Blumenthal R, Puri A. A novel class of photo-triggerable liposomes containing DPPC:DC ${ }_{8,9} \mathrm{PC}$ as vehicles for delivery of doxorubicin to cells. Biochim Biophys Acta. 2011; 1808: 117-126.

40. Khoobehi B, Char CA, Peyman GA, Schuele KM. Study of the mechanisms of laser-induced release of liposome-encapsulated dye. Lasers in Surgery and Medicine. 1990; 10: 303-309.

41. Khoobehi B, Peyman GA, Bhatt N, Moshfeghi D. Laser-induced experimental vascular occlusion using liposome-encapsulated ADP. Lasers in Surgery and Medicine. 1992; 12: 609-614.

42. VanderMeullen DL, Misra P, Michael J, Spears KG, Khoka AM. Laser mediated release of dye from liposomes. Photochem Photobiol. 1992; 56: 325-332.

43. Mordon S, Desmettre T, Devoisselle JM, Soulie S. Thermal damage assessment of blood vessels in a hamster skin flap model by fluorescence measurement of a liposome-dye system. Lasers in Surgery and Medicine. 1997; 20:131-141.

44. Mordon S, Devoisselle JM, Begu S, Desmettre T. Laser-induced release of liposome-encapsulated dye: A new diagnostic tool. Lasers Med Sci. 1998; 13:181-188.

45. Mackanos MA, Larabi M, Shinde R, Simanovskii DM, Guccione S, Contag CH. Laser-induced disruption of systemically administered liposomes for targeted drug delivery. J Biomed Optics. 2009; 14: 044009.

46. Dendramis KA, Allen PB, Reid PJ, Chiu DT. Spectrally tunable uncaging of biological stimuli from nanocapsules. Chem Commun. 2008;: 4795-4797.

47. Dendramis KA, Chiu DT. Laser photolysis of dye-sensitized nanocapsules occurs viaa photothermal pathway. J Am Chem Soc. 2009; 131: 16771-16778.

48. Gregersen KAD, Hill ZB, Gadd JC, Fujimoto BS, Maly DJ, Chiu DT. Intracellular delivery of bioactive molecules using light-addressable nanocapsules. ACS Nano 2010; 12: 7603-7611.

49. Paasonen L, Laaksonen T, Johans C, Yliperttula M, Kontturi K, Urtti A. Gold nanoparticles enable selective light-induced contentsrelease from liposomes. J Control Release. 2007; 122: 86-93.

50. An X, Zhang F, Zhub Y, Shena W. Photoinduced drug release from thermosensitive AuNPs-liposome using a AuNPs-switch. Chem Commun. 2010; 46: 7202-7204.

51. Mie G. Beiträgezur Optik trüber Medien, speziellkollodaler Metallösungen. Ann Phys. 1908; 330: 377-445.

52. Aden AL, Kerker M. Scattering of electromagnetic waves from two concentric spheres. J App Phys. 1951; 22: 1242-1246.

53. Oldenburg SJ, Averitt RD, Westcott SL, Halas NJ. Nanoengineering of optical resonances. Chem Phys Lett. 1998; 288: 243-247.

54. Troutman TS, Barton JK, Romanowski M. Biodegradable plasmon resonant nanoshells. Adv Mater. 2008; 20: 2604 - 2608

55. Ferrar T, O'Brien DF, Warshawsky A, Voycheck CL. Metalization of lipid vesicles via electroless plating. J Am Chem Soc. 1988; 110: 288-289.

56. Choi HS, Liu W, Misra P, Tanaka E, Zimmer JP, Ipe BI, Bawendi MG, Frangioni JV. Renal clearance of quantum dots. Nat Biotechnol. 2007; 25: 1165-1170.

57. Leung SJ, Kachur XM, Bobnick MC, Romanowski M. Wavelength-selective light-induced release from plasmon resonant liposomes. Adv Funct Mater. 2011; 21: 1113-1121.
58. Troutman TS, Leung SJ, Romanowski M. Light-induced content release from plasmon-resonant liposomes. Adv Mater. 2009; 21: 2334-2338.

59. Wu G, Mikhailovsky A, Khant HA, Fu C, Chiu W, Zasadzinski JA. Remotely triggered liposome release by near-infrared light absorption viahollow gold nanoshells. J Am Chem Soc. 2008;130: 8175-8177.

60. Volodkin DV, Skirtach AG, Möhwald H. Near-IR remote release from assemblies of liposomes and nanoparticles. Angew Chem Int Ed. 2009; 48: 1807-1809.

61. Anderson LJE, Hansen E, Lukianova-Hleb EY, Hafner JH, Lapotko DO. Optically guided controlled release from liposomes with tunable plasmonic nanobubbles. J Control Release. 2010; 144: 151-158.

62. Lukianova-Hleb E, Samaniego A, Wen J, Metelitsa L, Chang CC, Lapotko D. Selective gene transfection of individual cells in vitro with plasmonic nanobubbles. J Control Release. 2011; 152: 286-293.

63. Lukianova-Hleb E, Ren X, Zasadzinski JA, Wu X, Lapotko DO. Plasmonic nanobubbles enhance efficacy and selectivity of chemotherapy against drug-resistant cancer cells. Adv Mater. 2012; 24: 3831-3837.

64. Lukianova-Hleb E, Belyanin A, Kashinath S, Wu X, Lapotko D. Plasmonic nanobubble-enhanced endosomal escape processes for selective and guided intracellular delivery of chemotherapy to drug-resistant cancer cells. Biomaterials. 2012; 33: 1821-1826.

65. Jin Y, Gao X. Spectrally tunable leakage-free gold nanocontainers. J Am Chem Soc. 2009; 131: 17774-17776.

66. Wu C, Yu C, Chu M. A gold nanoshell with a silica inner shell synthesized using liposome templates for doxorubicin loading and near-infrared photothermal therapy. International J Nanomedicine. 2011; 6: 807-813.

67. Dave N, Liu J. Protection and promotion of UV radiation-induced liposome leakage via DNA-directed assembly with gold nanoparticles. Adv Mater. 2011; 23: 3182-3186.

68. Qin G, Li Z, Xia R, Li F, O'Neill BE, Goodwin JT, Khant HA, Chiu W, Li KC. Partially polymerized liposomes: Stable against leakage yet capable of instantaneous release for remote controlled drug delivery. Nanotechnology. 2011; 22: 155605.

69. Agarwal A, Mackey MA, El-Sayed MA, Bellamkonda RV. Remote triggered release of doxorubicin in tumors by synergistic application of thermosensitive liposomes and gold nanorods. ACS Nano. 2011; 5: 4919-4926.

70. Graff A, Winterhalter M, Meier W. Nanoreactors from polymer-stabilized liposomes. Langmuir. 2001; 17: 919-923.

71. Bolinger PY, Stamou D, Vogel H. Integrated nanoreactor systems: triggering the release and mixing of compounds inside single vesicles. J Am Chem Soc. 2004; 126: 8594-8595.

72. Kulin S, Kishore R, Helmerson K, Locascio L. Optical manipulation and fusion of liposomes as microreactors. Langmuir. 2003; 19: 8206-8210.

73. Wheeler AR, Throndset WR, Whelan RJ, Leach AM, Zare RN, Liao YH, Farrell K, Manger ID, Daridon A. Microfluidic device for single-cell analysis. Anal Chem. 2003; 75: 3581-3586.

74. Marchington RF, Arita Y, Tsampoula X, Gunn-Moore FJ, Dholakia K. Optical injection of mammalian cells using a microfluidic platform. Biomed Opt Exp. 2010; 1: 527-536.

75. Edwards KA, Bolduc OR, Baeumner AJ. Miniaturized bioanalytical systems: enhanced performance through liposomes. Curr Op Chem Biol. 2012; 16: 1-9.

76. Leung SJ, Romanowski M. Molecular catch and release: controlled delivery using optical trapping with light-responsive liposomes. Adv Mater. 2012; DOI: 10.1002/adma.201202180.

77. [Internet] Atlanta GA: American Cancer Society. Cancer facts and figures $2012 . \quad$ http://www.cancer.org/acs/groups/content/ @epidemiologysurveilance/documents/document/acspc-031941.pdf

78. Alberts B, Johnson A, Lewis J, Raff M, Roberts K, Walter P. Molecular Biology of the Cell; $5^{\text {th }}$ edition. New York: Garland Science. 2008

79. Weissleder R, Ntziachristos V. Shedding light onto live molecular targets. Nature Medicine. 2003; 9: 123-128.

80. Mehlen P, Puisieux A. Metastasis: a question of life or death. Nature. 2006; 6: 449-458.

81. Calvo F, Sahai E. Cell communication networks in cancer invasion. Curr Op Cell Biol. 2011; 23: 621-629.

82. Ingber D E. Can cancer be reversed by engineering the tumor microenvironment? Semin Cancer Biol. 2008; 18: 356-364. 